\title{
Structure of mycobacterial $\mathrm{CIII}_{2} \mathrm{CIV}_{2}$ respiratory supercomplex bound to the tuberculosis drug candidate telacebec $(\mathbf{Q 2 0 3})$
}

David J. Yanofsky ${ }^{1,2,6}$, Justin M. Di Trani ${ }^{1,6}$, Sylwia Krol ${ }^{3}$, Rana Abdelaziz ${ }^{4}$, Stephanie A. Bueler $^{1}$, Peter Imming ${ }^{4}$, Peter Brzezinski ${ }^{3}$, John L. Rubinstein ${ }^{1,2,5}$

1. Molecular Medicine Program, The Hospital for Sick Children, Toronto, Ontario, Canada.

2. Department of Medical Biophysics, The University of Toronto, Toronto, Ontario, Canada.

3. Department of Biochemistry and Biophysics, Arrhenius Laboratories for Natural Science, Stockholm University, Stockholm, Sweden

4. Department of Pharmaceutical/Medicinal Chemistry and Clinical Pharmacy, Faculty of Natural

Sciences, Martin-Luther-Universitaet, Halle-Wittenberg, 06120 Halle (Saale), Germany

5. Department of Biochemistry, The University of Toronto, Toronto, Ontario, Canada

6. These authors contributed equally: David J. Yanofsky, Justin M. Di Trani

Correspondence: peter.imming@pharmazie.uni-halle.de, peterb@dbb.su.se,

john.rubinstein@utoronto.ca

\begin{abstract}
The imidazopyridine telacebec, also known as Q203, is one of only a few new classes of compounds in more than fifty years with demonstrated antituberculosis activity in humans. Telacebec inhibits the mycobacterial respiratory supercomplex composed of complexes III and IV $\left(\mathrm{CIII}_{2} \mathrm{CIV}_{2}\right)$. In mycobacterial electron transport chains, $\mathrm{CIII}_{2} \mathrm{CIV}_{2}$ replaces canonical CIII and CIV, transferring electrons from the intermediate carrier menaquinol to the final acceptor, molecular oxygen, while simultaneously transferring protons across the inner membrane to power ATP synthesis. We show that telacebec inhibits the menaquinol:oxygen oxidoreductase activity of purified Mycobacterium smegmatis $\mathrm{CIII}_{2} \mathrm{CIV}_{2}$ at concentrations similar to those needed to inhibit electron transfer in mycobacterial membranes and Mycobacterium tuberculosis growth in culture. We then used electron cryomicroscopy (cryoEM) to determine structures of $\mathrm{CIII}_{2} \mathrm{CIV}_{2}$ both in the presence and absence of telacebec. The structures suggest that telacebec prevents menaquinol oxidation by blocking two different menaquinol binding modes to prevent $\mathrm{CIII}_{2} \mathrm{CIV}_{2}$ activity.
\end{abstract}

\section{Introduction}

Numerous bacteria from the strictly-aerobic genus Mycobacterium are human pathogens. In particular, infection by Mycobacterium tuberculosis and closely related species result in the disease tuberculosis (TB). In most years TB is the leading cause of death by infectious disease internationally, with an increasing incidence of drug-resistant infections (Global Tuberculosis Report, 2020). Nontuberculosis mycobacterial pathogens include M. leprae, which causes leprosy, M. ulcerans, which causes Buruli ulcer, and M. avium and M. abscessus, which infect immunocompromised and cystic fibrosis patients, respectively. The discovery of bedaquiline from a phenotypic screen with non-pathogenic $M$. smegmatis, and its subsequent development into an effective therapeutic, has revolutionized the treatment of multidrug-resistant and extensively drug-resistant TB (Global Tuberculosis Report, 2020; World Health Organization, 2019). Bedaquiline binds the membrane region of mycobacterial ATP synthase (Andries et al., 2005; Guo et al., 2021; Preiss et al., 2015), blocking proton translocation and ATP synthesis. 
Thus, in addition to providing an invaluable therapeutic tool, bedaquiline established oxidative phosphorylation as a target space for antibiotics against mycobacteria. Subsequent to the discovery of bedaquiline, numerous compounds have been identified that target either ATP synthase or the electron transport chain complexes that establish the transmembrane proton motive force (PMF) that drives ATP synthesis (Cook et al., 2017).

In canonical mitochondrial electron transport chains, Complexes I and II oxidize NADH (the reduced form of nicotinamide adenine dinucleotide) and succinate, respectively. The electrons from these substrates are used to reduce a membrane-bound pool of ubiquinone (UQ) to ubiquinol $\left(\mathrm{UQH}_{2}\right)$. Electrons from $\mathrm{UQH}_{2}$ are then passed successively to CIII (also known as cytochrome [cyt.] $b c_{1}$,), soluble cyt. $c$, and CIV (also known as cyt. $c$ oxidase or cyt. $a a_{3}$ ) before ultimately reducing molecular oxygen to water. Complexes I, III, and IV couple electron transfer to proton transfer across the membrane, thereby generating the PMF that drives ATP synthesis. In contrast to mitochondria and many bacteria, mycobacteria possess a branched electron transport chain (Reviewed in (Cook et al., 2017)). Rather than UQ, mycobacterial respiration relies on menaquinone (MQ) as an intermediate electron carrier. $\mathrm{MQH}_{2}$ can reduce molecular oxygen via two $\mathrm{MQH}_{2}: \mathrm{O}_{2}$ oxidoreductases: cyt. $b d$ and cyt. $b c c-a a_{3}$, the latter being equivalent to a combination of canonical CIII and CIV with the stoichiometry $\mathrm{CIII}_{2} \mathrm{CIV}_{2}$. Bioenergetically, the $\mathrm{CIII}_{2} \mathrm{CIV}_{2}$ supercomplex and cyt. $b d$ branches of the mycobacterial electron transport chain are not equivalent, with $\mathrm{CIII}_{2} \mathrm{CIV}_{2}$ transferring three protons across the membrane for each electron used to reduce $\mathrm{O}_{2}$ while cyt. $b d$ transfers only one proton across the membrane for each electron. However, despite this difference, enzyme utilization in mycobacterial electron transport chains can adapt to changes in environmental conditions and treatment with respiratory complex inhibitors (Arora et al., 2014; Berney and Cook, 2010), which complicates targeting of respiration by antimycobacterial drugs (Beites et al., 2019).

Structural analysis of the $\mathrm{CIII}_{2} \mathrm{CIV}_{2}$ supercomplex from $M$. smegmatis led to the discovery that a dimeric type-C superoxide dismutase (SOD) is an integral component of the assembly (Gong et al., 2018; Wiseman et al., 2018). The SOD dimer is found on the periplasmic side of $\mathrm{CIII}_{2} \mathrm{CIV}_{2}$ and is held in place by its two N-terminal tails, which bind to the complex's QcrB subunits. Both QcrB and the SOD subunit are highly conserved between M. smegmatis and M. tuberculosis, with $99.6 \%$ and $95 \%$ sequence similarity and $81.1 \%$ and $64.8 \%$ sequence identity, respectively, suggesting that a similar association occurs in the M. tuberculosis enzyme. M. tuberculosis mutants that lack SOD are susceptible to killing within macrophages (Piddington et al., 2001), emphasizing the importance of the subunit. Given its position within $\mathrm{CIII}_{2} \mathrm{CIV}_{2}$, it is possible that the SOD subunit abstracts electrons from reactive oxygen species formed during respiration or generated by host-defense mechanisms in the phagolysosome upon phagocytosis of $M$. tuberculosis and directs them through the respiratory chain to contribute to the PMF and ATP synthesis (Gong et al., 2018; Wiseman et al., 2018).

Although killing of $M$. tuberculosis with electron transport chain inhibitors may require simultaneously blocking both the $\mathrm{CIII}_{2} \mathrm{CIV}_{2}$ and cyt. $b d$ branches for oxygen reduction (Arora et al., 2014; Beites et al., 2019; Matsoso et al., 2005), high-profile candidate TB therapeutics have been identified that bind to CIII within $\mathrm{CIII}_{2} \mathrm{CIV}_{2}$ (Pethe et al., 2013; Rybniker et al., 2015). 
Similarly, while $\mathrm{CIII}_{2} \mathrm{CIV}_{2}$ is not essential in $M$. smegmatis, its disruption causes severe growth defects (Matsoso et al., 2005). In contrast, $\mathrm{CIII}_{2} \mathrm{CIV}_{2}$ is essential in M. leprae and M. ulcerans, which lack the cyt. $b d$ oxidase branch of the electron transport chain entirely (Cole et al., 2001; Demangel et al., 2009).

Rather than pumping protons, CIII contributes to the PMF by separating positive and negative charges across the membrane through a mechanism known as the Q cycle (reviewed in (Sarewicz and Osyczka, 2015; Xia et al., 2013). Each CIII contains two sites where redox reactions with MQ occur: a QP site near the positive (periplasmic) side of the membrane where $\mathrm{MQH}_{2}$ is oxidized and a $Q_{N}$ site near the negative (cytoplasmic) side of the membrane where MQ is reduced. In the current understanding of the $\mathrm{Q}$ cycle in mycobacteria, oxidation of $\mathrm{MQH}_{2}$ in the Qp site leads to release of two protons to the positive side of the membrane. The first electron from this oxidation reaction is passed to a [2Fe-2S] Rieske center $(\mathrm{FeS})$ in subunit QcrA where it consecutively reduces the cyt. $c c$ domain of the $\mathrm{QcrC}$ subunit and the $\mathrm{Cu}_{\mathrm{A}}$ di-nuclear center of CIV. The second electron from the $\mathrm{MQH}_{2}$ in the QP site is transferred first to heme $b_{\mathrm{L}}$ and then heme $b_{\mathrm{H}}$, both in subunit QcrB of CIII, before reducing a MQ molecule in the QN site to MQ ${ }^{\bullet}$. Oxidation of a second $\mathrm{MQH}_{2}$ in the $\mathrm{QP}_{\mathrm{P}}$ site and repetition of this series of events leads to reduction of $\mathrm{MQ}^{\bullet-}$ to $\mathrm{MQH}_{2}$ in the $\mathrm{Q}_{\mathrm{N}}$ site, upon abstraction of two protons from the negative side of the membrane, thereby contributing to the PMF and the pool of reduced $\mathrm{MQH}_{2}$ in the membrane. Within CIV, electrons are transferred from the $\mathrm{Cu}_{\mathrm{A}}$ di-nuclear center to the heme $a_{3}$ $\mathrm{Cu}_{\mathrm{B}}$ binuclear catalytic site via heme $a$, driving proton pumping across the membrane.

Telacebec (also known as Q203), was identified in a screen of macrophages infected with $M$. tuberculosis (Pethe et al., 2013). Generation of resistance mutants bearing T313I and T313A mutations in the $q c r B$ gene indicated that telacebec targets $\mathrm{CIII}$ of the $\mathrm{CIII}_{2} \mathrm{CIV}_{2}$ supercomplex. A recent Phase 2 clinical trial demonstrated a decrease in viable mycobacterial sputum load with increasing dose of telacebec, supporting further development and making telacebec one of only a few new drug classes in more than fifty years with demonstrated antituberculosis activity in humans (van Niekerk et al., 2020). Telacebec may also have clinical utility in treating nontuberculosis mycobacterial infections, such as Buruli ulcer (Almeida et al., 2020; Van Der Werf et al., 2020).

Here we use electron cryomicroscopy (cryoEM) to investigate how telacebec inhibits purified $\mathrm{CIII}_{2} \mathrm{CIV}_{2}$ from M. smegmatis. We develop conditions for $\mathrm{CIII}_{2} \mathrm{CIV}_{2}$ activity assays that limit the spontaneous autoxidation of $\mathrm{MQH}_{2}$ analogues that has hampered previous analysis of $\mathrm{MQH}_{2}: \mathrm{O}_{2}$ oxidoreductase activity with purified $\mathrm{CIII}_{2} \mathrm{CIV}_{2}$. The assays show that telacebec inhibits $\mathrm{CIII}_{2} \mathrm{CIV}_{2}$ activity at concentrations comparable to those that inhibit electron transfer in mycobacterial membranes. CryoEM of $\mathrm{CIII}_{2} \mathrm{CIV}_{2}$ demonstrates both the presence of the LpqE subunit (Gong et al., 2018) and different conformations of the cyt. cc domain (Wiseman et al., 2018), which have previously been observed separately but not together. $3 \mathrm{D}$ variability analysis of the structure shows that the SOD subunit can move toward cyt. $c c$, supporting the possibility of direct electron transfer from superoxide to CIV . CryoEM of the $\mathrm{CIII}_{2} \mathrm{CIV}_{2}$ :telacebec complex allows localization of the telacebec binding site with the imidazopyridine moiety and A-benzene ring of telacebec forming most protein-inhibitor contacts. 


\section{Results}

Structure of $\mathrm{CIII}_{2} \mathrm{CIV}_{2}$ reveals movement of SOD subunit and cyt. cc domain In order facilitate isolation of $\mathrm{CIII}_{2} \mathrm{CIV}_{2}$, we used the ORBIT (oligonucleotide-mediated recombineering followed by Bxb1 integrase) strategy (Murphy et al., 2018) to introduce

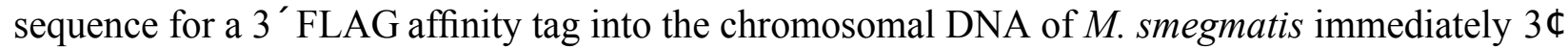
to the $q c r B$ gene. While M. smegmatis is typically grown in $7 \mathrm{H} 9$ medium supplemented with albumin, dextrose, and sodium chloride (ADS), we found that supplementing instead with tryptone, dextrose, and sodium chloride (TDS), which is more economical for large scale culture, gave equivalent or superior growth. Purification of $\mathrm{CIII}_{2} \mathrm{CIV}_{2}$ from $M$. smegmatis grown in these conditions gave a high yield of enzyme with clear bands for most of the known subunits of the complex (Fig. 1A). We observed that following affinity purification, gel filtration chromatography of the enzyme led to depletion of the LpqE and SOD subunits (Fig. 1A, right) compared to affinity purification alone (Fig. 1A, left), and consequently this purification step was avoided.

CryoEM of the $\mathrm{CIII}_{2} \mathrm{CIV}_{2}$ preparation allowed for calculation of a 3D map of the enzyme at a nominal resolution of $3.0 \AA$ (Fig. 1B, Figure 1 - Figure Supplement 1, and Table 1). The map shows strong density for the LpqE subunit (Fig. 1B, orange). LpqE was observed in one previous structural study of $\mathrm{CIII}_{2} \mathrm{CIV}_{2}$ from M. smegmatis (Gong et al., 2018) but was absent in another (Wiseman et al., 2018), presumably due to depletion of the subunit during purification of the supercomplex. In the structure missing LpqE, the cyt. $c c$ domain of subunit QcrC adopts both an 'open' and a 'closed' conformation, while the structure with LpqE was found only in the closed conformation. The closed conformation creates a direct electronic connection between heme $c_{\mathrm{II}}$ of CIII and $\mathrm{Cu}_{\mathrm{A}}$ of CIV (Gong et al., 2018; Wiseman et al., 2018). In the open conformation, heme $c_{\text {II }}$ from the cyt. $c c$ domain is too far from $\mathrm{Cu}_{\mathrm{A}}$ to allow electron transfer, leading to the hypothesis that switching between the closed and open conformations plays a role in controlling the flow of electrons through the supercomplex (Wiseman et al., 2018). In contrast, LpqE was hypothesized to strengthen the physical attachment between CIII and CIV (Gong et al., 2018). 3D variability analysis with the current dataset (Punjani and Fleet, 2021), focused on one half of the supercomplex, revealed complexes with and without LpqE. Where LpqE was missing, the cyt. $c c$ domain exhibits the open conformation, while complexes with LpqE show only the closed conformation of cyt. $c c$ (Figure 1 - Figure Supplement 2). Clashes between LpqE and the open conformation of cyt. $c c$ suggest that LpqE prevents the open conformation.

In previous studies the SOD subunit of $\mathrm{CIII}_{2} \mathrm{CIV}_{2}$ was poorly resolved in cryoEM maps and appeared as a diffuse density (Gong et al., 2018; Wiseman et al., 2018). In the present map the overall shape of SOD, although still at lower density than the rest of the complex, was more readily apparent (Fig. 1B, pink). The N-terminal anchors from the SOD dimer that bind to subunit QcrB are well resolved and terminate at the middle of the complex with a lipid anchor (Fig. 1B, beige). The improved density for the SOD subunit allowed fitting of a homology model of the protein into the map with a slight rotation relative to how it was fit previously (Fig. 1C, right). 3D variability analysis (Fig. 1C) shows that SOD moves between the center of the complex, where it was observed previously (Gong et al., 2018; Wiseman et al., 2018) to immediately above heme $c_{\text {I }}$. This proximity suggests that SOD may indeed transfer electrons abstracted from superoxide in the periplasm of $M$. smegmatis to CIV thereby contributing to the 
PMF (Gong et al., 2018; Wiseman et al., 2018), although this hypothesis requires further testing. The overall resolution of the map, which is somewhat better than in previous studies, allowed refinement of an atomic model for $\mathrm{CIII}_{2} \mathrm{CIV}_{2}$ including residues T82, E83, A123 to D131, and S183 from LpqE, and residues H57 to G78 from MSMEG_4693 (also known as CtaJ), which could not be modelled previously (Fig. 1D, Fig. 1 - Figure Supplement 3, and Table 1). The model shown in Fig. 1D illustrates one cyt. $c c$ domain in the closed position with LpqE present (Fig. 1D, right side: cyan and orange) and the other cyt. $c c$ domain in the open position without LpqE (Fig. 1D, left side: cyan).

Nanomolar telacebec inhibits oxidoreductase activity with purified $\mathrm{CIII}_{2} \mathrm{CIV}_{2}$ To investigate inhibition of $\mathrm{CIII}_{2} \mathrm{CIV}_{2}$ by telacebec, we established a supercomplex activity assay, based on measurement of oxygen consumption with a Clark-type electrode. The mycobacterial electron transport chain uses $\mathrm{MQH}_{2}$ as the electron donor for $\mathrm{CIII}_{2} \mathrm{CIV}_{2}$ while in canonical mitochondrial electron transport chains $\mathrm{UQH}_{2}$ donates electrons to $\mathrm{CIII}_{2}$ (Cook et al., 2017). Both $\mathrm{UQH}_{2}$ and $\mathrm{MQH}_{2}$ are insoluble in aqueous solution and consequently soluble analogues must be employed as substrates in assays with detergent-solubilized enzymes. The midpoint potentials of the redox sites of mycobacterial $\mathrm{CIII}_{2}$ are lower than those of canonical mitochondrial $\mathrm{CIII}_{2}$ (Kao et al., 2016), and as a result $\mathrm{UQH}_{2}$ analogues typically used in $\mathrm{CIII}_{2}$ assays are not able to reduce $\mathrm{CIII}_{2}$ of the $M$. smegmatis supercomplex. $\mathrm{MQH}_{2}$ analogues capable of reducing $\mathrm{CIII}_{2} \mathrm{CIV}_{2}$ suffer from autoxidation at neutral or basic $\mathrm{pH}$, which leads to oxygen reduction even in the absence of enzyme. This background oxygen-reduction rate is typically subtracted from that observed in the presence of enzyme to calculate the enzyme-catalyzed oxidoreductase activity. Previous measurement of inhibition of $\mathrm{CIII}_{2} \mathrm{CIV}_{2}$ by telacebec employed menadiol as the electron donor, which is highly soluble in aqueous solution, but rapidly autoxidizes, leading to the background signal from autoxidation in the absence of enzyme being higher than the signal from oxygen reduction by the enzyme (Gong et al., 2018). Instead, we selected 2,3-dimethyl-[1,4]naphthoquinone (DMW), which we chemically reduced to $\mathrm{DMWH}_{2}$, as the substrate for oxygen reduction assays (Graf et al., 2016; Wiseman et al., 2018). Initial activity assays led to anomalous results where addition of low concentrations of $\mathrm{CIII}_{2} \mathrm{CIV}_{2}$ to the assay mixture appeared to decrease the rate of oxygen reduction below the background autoxidation rate. On subsequent investigation, we realized that at low concentrations of $\mathrm{CIII}_{2} \mathrm{CIV}_{2}$ the SOD subunit suppresses autoxidation of DMW more than $\mathrm{CIII}_{2} \mathrm{CIV}_{2}$ catalyzes oxidation of DMW. This suppression of QH2 autoxidation by SOD, which has been described previously (Cadenas et al., 1988), can lead to apparent negative activities for $\mathrm{CIII}_{2} \mathrm{CIV}_{2}$ when the background autoxidation is subtracted. To remove this source of error, we established that bovine C-type SOD can similarly limit the autoxidation of DMW (Fig. 2A, blue and orange curves). Thus, by adding an excess of exogenous bovine SOD to assays, the $\mathrm{CIII}_{2} \mathrm{CIV}_{2}$ 's DMW: $\mathrm{O}_{2}$ oxidoreductase activity can be measured with suppression of DMW autoxidation (e.g. Fig. 2A, green). With $500 \mathrm{nM}$ SOD added, the $\mathrm{CIII}_{2} \mathrm{CIV}_{2}$ 's $\mathrm{DMW}: \mathrm{O}_{2}$ oxidoreductase activity was measured at $91 \pm 4 \mathrm{e}^{-/ \mathrm{s}}$ ( \pm s.d., $\mathrm{n}=6$ independent assays), which is nearly an order of magnitude greater than the apparent activity found previously (Wiseman et al., 2018). We subsequently added $500 \mathrm{nM}$ bovine SOD to all assays to limit autoxidation of DMW. 
Telacebec (Fig. 2B) is a potent inhibitor of mycobacterial $\mathrm{CIII}_{2}$ (Pethe et al., 2013). The compound consists of an imidazo[1,2-a]pyridine attached via an amide linker to a N-[(4-\{4-[4(trifluoromethoxy)phenyl]piperidin-1-yl \} phenyl)methyl] 'tail'. Titrations of $\mathrm{CIII}_{2} \mathrm{CIV}_{2}$ activity with varying concentrations of telacebec (Fig. 2C) show an $\mathrm{IC}_{50}$ of $53 \mathrm{nM} \pm 19( \pm$ s.d., $\mathrm{n}=3$ independent titrations) with $65 \mathrm{nM} \mathrm{CIII}_{2} \mathrm{CIV}_{2}$ and $100 \mu \mathrm{M}$ DMW. This $\mathrm{IC}_{50}$ is lower than the $840 \pm 22 \mathrm{nM}$ seen with the menadiol based assay (Gong et al., 2018), but higher than the $20 \mathrm{nM}$ concentration needed to inhibit $50 \%$ of respiratory chain activity with inverted membrane vesicles from $M$. smegmatis (Lu et al., 2018) or $2.7 \mathrm{nM}$ required to inhibit the $50 \%$ of $M$. tuberculosis growth in liquid culture (Pethe et al., 2013).

The M. tuberculosis telacebec resistance mutations T313A and T313I (Pethe et al., 2013), equivalent to mutation of Thr308 in M. smegmatis, are near the QP site and suggest that the inhibitor could interfere with MQ binding to $\mathrm{CIII}_{2} \mathrm{CIV}_{2}$. The increased $\mathrm{IC}_{50}$ in the current assay compared to assays with inverted membrane vesicles or bacterial growth in liquid culture may be due to the binding affinity or high concentration of DMW, which could allow DMW to compete with telacebec for binding to the complex.

The $\mathrm{CIII}_{2} \mathrm{CIV}_{2}$ structure has endogenous $\mathrm{MQ}$ in its $Q_{P}$ site

As telacebec is expected to bind near the QP site of $\mathrm{CIII}_{2} \mathrm{CIV}_{2}$, we carefully characterized this site in the cryoEM map of the enzyme in the absence of inhibitor. The Qp site is near the periplasmic side of the membrane, located between heme $b_{\mathrm{L}}$ and the FeS cluster (Fig. 3A), and is formed by several loops and ahelices from both the QcrB and QcrA subunits (Fig. 3B). The arrangement of structural elements in the site is conserved from other CIIIs (Sarewicz and Osyczka, 2015). The entrance to the $\mathrm{Q}_{\mathrm{p}}$ site is formed by the $\mathrm{C}$ and $\mathrm{F}$ transmembrane ahelices, and the cd2 ahelix that separates the periplasmic side of the QP pocket from the QcrA subunit. The ef helix and ef loop from the QcrB subunit are deeper in the Qp site, as is a short section from the QcrA subunit that includes the FeS-bound His368 residue (Fig. 3B).

In the inhibitor-free structure there is strong density for endogenous MQ in the Qp site (Fig. 3B, pale blue surface). The naphthoquinone head group of MQ is positioned near the entrance to the site, between the $\mathrm{F}$ and $\mathrm{C}$ helices, with the MQ tail trailing towards the $\mathrm{Q}_{\mathrm{N}}$ site (Fig. 3A). This position for endogenous MQ was reported in a previous study of $\mathrm{CIII}_{2} \mathrm{CIV}_{2}$ from M. smegmatis (Gong et al., 2018). In this position, the naphthoquinone head group is $\sim 14 \AA$ away from the FeS cluster and the hydroxyl proton is $\sim 15 \AA$ from His 368 , which is too far for rapid coupled electron and proton transfer from $\mathrm{MQH}_{2}$ to $\mathrm{FeS}$ and His368, respectively. This distance contrasts the deeper binding position adopted by UQ in ovine $\mathrm{CIII}_{2}$ (Letts et al., 2019). It is also further from the FeS than the position observed for UQ-analogue inhibitors such as stigmatellin bound to chicken $\mathrm{CIII}_{2}$ (Zhang et al., 1998), as well as 5-undecyl-6-hydroxy-4,7-dioxobenzothiazole (Esser et al., 2004) and 2-n-nonyl-4-hydroxyquinoline N-oxide (Gao et al., 2003) bound to bovine $\mathrm{CIII}_{2}$. In the deeper position the head groups of UQ or its analogues are wedged between the ef helix/loop, and the cd2 helix, with the tail trailing between the F and $\mathrm{C}$ helix at the Qp site entrance (Fig. 3 - Figure Supplement 1A). In these structures, the distance between the quinone head group and the FeS cluster depends on the position of the mobile Rieske head domain but can be as little as $\sim 7 \AA$, allowing for rapid electron transfer from the $\mathrm{UQH}_{2}$ to the FeS (Moser et al., 2006). 
Telacebec replaces $\mathrm{MQ}$ in the $Q_{P}$ site of active $\mathrm{CIII}_{2} \mathrm{CIV}_{2}$

Our initial attempts to image $\mathrm{CIII}_{2} \mathrm{CIV}_{2}$ with telacebec failed to resolve the inhibitor (Fig. 3 Figure Supplement 1B, left), leading us to consider the possibility that inhibitor binding occurs during substrate turnover by the enzyme. CryoEM of $\mathrm{CIII}_{2} \mathrm{CIV}_{2}$ in the presence of $\mathrm{DMWH}_{2}$ but without telacebec confirmed that under these conditions the density in the Qp site was indistinguishable from MQ seen with the enzyme at rest (Fig. 3 - Figure Supplement 1B, right). We then incubated $\mathrm{CIII}_{2} \mathrm{CIV}_{2}$ with both $\mathrm{DMWH}_{2}$ and telacebec to produce an inhibited complex and determined the structure of this complex to a nominal resolution of $3.0 \AA$ by cryoEM (Fig. 1 - Figure Supplement 1, Table S1). Telacebec binding did not cause large-scale conformational changes in $\mathrm{CIII}_{2} \mathrm{CIV}_{2}$ but produced a clear density for the inhibitor in each of the two Qp sites in the $\mathrm{CIII}_{2}$ dimer (Fig. 3C). The inhibitor's imidazopyridine moiety, amide linker region, and A-phenyl and B-piperidinyl moieties are all resolved clearly, with weaker density toward the end of the tail, which points into the lipid bilayer toward the cytoplasmic side of the membrane (Fig. 3C, pale blue surface). These densities show that telacebec binds with its head group deep within the QP binding pocket in a pose similar to UQ and the UQ-analogue inhibitors bound within the canonical $\mathrm{CIII}_{2}$ as described above (Fig. 3 - Figure Supplement 1A). Telacebec's imidazopyridine moiety forms multiple interactions with the protein to stabilize inhibitor binding. Although hydrogen bonds cannot be detected with complete confidence at the present resolution, the position of the N1 nitrogen in telacebec's imidazopyridine moiety is consistent with formation of a hydrogen bond with the His368 from the QcrA subunit, which also binds the FeS group (Fig. 3C and D, dashed teal line). The occurrence of a similar hydrogen bond between UQ and the equivalent histidine residue in canonical $\mathrm{CIII}_{2}$ (Zhang et al., 1998) has been proposed to coordinate the Q cycle (e.g. see (Sarewicz and Osyczka, 2015)). The 2-ethyl group from the imidazopyridine is $\sim 4 \AA$ away from Ile178 from the QcrB subunit, providing hydrophobic interactions (Fig. 3C and D, dashed green line), while the 6-chloro group from the imidazopyridine is close to the backbone carboxyl group of Leu166 from the QcrB subunit, enabling formation of a possible halogen bond (Fig. 3C and D, dashed purple line).

In addition to its imidazopyridine moiety, the amide linker and tail of telacebec also contact subunit QcrB, stabilizing binding. Thr308, which is homologous with M. tuberculosis Thr313 and is known to be important for binding (Pethe et al., 2013), is $<4 \AA$ away from the linker region of telacebec. Although the rotameric state of Thr308 is ambiguous at the current resolution, one of the rotamer states could form a stabilizing hydrogen bond with the carbonyl group of the linker region (Fig. 3C and D, dashed teal line). Finally, Phe156 is $\sim 3.5 \AA$ from the A-phenyl group of telacebec, allowing for aromatic-aromatic interaction between the protein and inhibitor (Burley and Petsko, 1985) (Fig. 3C and D, dashed pink line) Interestingly, in the inhibitor-free specimen, the MQ head group is positioned similarly to the A-phenyl ring of telacebec and may form similar stabilizing contacts with the QcrB subunit (Fig. 3B and C).

Telacebec blocks both the standby and oxidation positions for $M Q$

The telacebec-bound structure also provides insight into the basic mechanism of MQ: $\mathrm{O}_{2}$ oxidoreductase activity by $\mathrm{CIII}_{2} \mathrm{CIV}_{2}$. Multiple MQ binding sites were modelled in an earlier 3.5 $\AA$ resolution cryoEM density map of $\mathrm{CIII}_{2} \mathrm{CIV}_{2}$, but the significance of these sites was not clear (Gong et al., 2018). The structure presented here shows that telacebec, which can inhibit MQ: $\mathrm{O}_{2}$ 
oxidoreductase activity completely (Fig. 2C), binds only at the Qp site. Therefore, it is unlikely that MQ-binding other than within the Qp site is involved in electron transfer to oxygen. During the $\mathrm{Q}$ cycle, two molecules of $\mathrm{MQH}_{2}$ are oxidized to $\mathrm{MQ}$ at the $\mathrm{Q}_{\mathrm{P}}$ site for each molecule of $\mathrm{MQ}$ reduced to $\mathrm{MQH}_{2}$ at the $\mathrm{Q}_{\mathrm{N}}$ site. It is possible that $\mathrm{MQ}$ is channeled between the $\mathrm{Q}_{\mathrm{P}}$ and $\mathrm{Q}_{\mathrm{N}}$ sites by staying loosely bound to the supercomplex surface, with the additional MQ sites serving as intermediate positions along the channeling pathway. A similar model was suggested for the spinach $b_{6} f$ complex, which is structurally and functionally related to CIII and carries out the Q cycle in plant chloroplasts with the hydrophobic electron carrier plastiquinone (Malone et al., 2019). It is also possible that these alternative MQ sites sequester MQ, increasing the local concentration of substrate near its two binding sites, which has been seen to increase the local concentration of ligands in other systems (Vauquelin and Charlton, 2010).

Within the Qp site different positions for both UQ and MQ have been described previously, both for canonical CIII and for CIII within a $\mathrm{CIII}_{2} \mathrm{CIV}_{2}$ supercomplex, respectively (Ding et al., 1992; Moe et al., 2021). As discussed above, in the present structure, the endogenous MQ is too far for rapid transfer of protons and electrons from $\mathrm{MQH}_{2}$ to His368 and the FeS center, respectively (Fig. 4A). This binding site within $Q_{p}$ is known as the Q1b position. In contrast, movement of MQ deeper within the Qp site to the Q1a position would bring it less than $10 \AA$ from FeS, close enough to donate electrons to the redox center and protons to His368 (Fig. 4B). In an emerging model for $\mathrm{CIII}_{2} \mathrm{CIV}_{2}$ function, the Q1b position serves as a "stand-by" site for MQ, with oxidation of the substrate occurring only upon relocation to Q1a (see also (Moe et al., 2021; Mulkidjanian, 2005)). The structure of $\mathrm{CIII}_{2} \mathrm{CIV}_{2}$ with telacebec bound shows how that the compound serves as dual site inhibitor (Fig. 4C), with the imidazopyridine group bound to the Q1a position and A-phenyl portion of the tail bound to the Q1b position. Indeed, the possible hydrogen bond between Thr308 and the carboxyl group in the linker region of telacebec could also occur between Thr308 and MQ, stabilizing it in the Q1b position. Therefore, the observed pose of the inhibitor within the enzyme not only blocks MQ access to the FeS center but fills the QP site entirely.

Inhibiting $\mathrm{CIII}_{2} \mathrm{CIV}_{2}$ has demonstrated antituberculosis activity in humans (van Niekerk et al., 2020), even though $M$. tuberculosis possesses cyt. $b d$ as an alternative enzyme that can oxidize $\mathrm{MQH}_{2}$ and sustain the electron flux to oxygen in the mycobacterial membrane. More potent killing of mycobacterial pathogens can be accomplished by simultaneous inhibition of both the $\mathrm{CIII}_{2} \mathrm{CIV}_{2}$ and cyt. $b d$ terminal oxidases (Beites et al., 2019). The present study demonstrates how cryoEM can reveal the mechanisms of electron transport chain inhibitors, enabling new strategies for targeting mycobacterial infections.

\section{Methods}

\section{Construction of $M$. smegmatis strain, cell culture, and protein isolation}

An M. smegmatis strain with a $3 \times$ FLAG tag at the $C$ terminus of subunit QcrB was generated with the oligonucleotide-mediated recombineering followed by Bxb integrase targeting (ORBIT) method (Murphy et al., 2018). This method requires transformation of the parent strain with a plasmid encoding the Che9c phage RecT annealase and Bxb1 integrase, a payload plasmid with the desired insert, and an oligonucleotide that guides integration of the payload into the chromosomal DNA. The parent strain MC2155 was transformed with plasmid pKM444, which encodes the Che9c annealase and Bxb1 integrase. The resulting strain was subsequently 
transformed with payload plasmid pSAB41, which encodes a $3 \times$ FLAG tag and was described previously (Guo et al., 2021), as well as the targeting oligonucleotide 5'-

CAAGTCGCTCACGGCGCTCAAGGAGCACCAGGACCGCATCCACGGCAACGGGGAGA CCAACGGTCATCACGGTTTGTCTGGTCAACCACCGCGGTCTCAGTGGTGTACGGTAC AAACCTGATCGCTGAGATACTCGGATCGCCGCAATTCCTCTTCGGAGGGGTTGCGGC GATCTTTTTATGTGCGCTTTC-3'. The resulting strain “M. smegmatis QcrB-3xFLAG” was selected with hygromycin $(50 \mu \mathrm{g} / \mathrm{ml})$ and correct insertion of the $3 \times$ FLAG sequence was confirmed by colony PCR.

M. smegmatis was cultured in 7H9 medium (Sigma) supplemented with TDS (10 g/L tryptone, 2 $\mathrm{g} / \mathrm{L}$ dextrose, $0.8 \mathrm{~g} / \mathrm{L} \mathrm{NaCl})$. A preculture in liquid medium $(15 \mathrm{~mL})$ was inoculated with cells from an agar plate and grown at $30{ }^{\circ} \mathrm{C}$ with shaking at $180 \mathrm{rpm}$ for $48 \mathrm{~h}$. This culture was used to inoculate a larger culture $(6 \mathrm{~L})$, which was grown at the same conditions for a further $48 \mathrm{~h}$. Cells were harvested by centrifugation at $6,900 \times \mathrm{g}$ for $20 \mathrm{~min}$ and frozen in liquid nitrogen for subsequent use. After thawing, cells were broken by four passes through a continuous flow cell disruptor (Avestin) at $20 \mathrm{kpsi}$ and membranes were harvested by centrifugation at $39,000 \times \mathrm{g}$ for $30 \mathrm{~min}$.

To purify $\mathrm{CIII}_{2} \mathrm{CIV}_{2}$, membranes were resuspended in lysis buffer (50 mM Tris-HCl pH 7.5, 100 $\mathrm{mM} \mathrm{NaCl}, 0.5 \mathrm{mM}$ EDTA) at $4 \mathrm{ml} / \mathrm{g}$ and solid dodecyl maltoside (DDM) detergent was added to $1 \%(\mathrm{w} / \mathrm{v})$ with stirring at $4{ }^{\circ} \mathrm{C}$ for $45 \mathrm{~min}$. Insoluble material was removed by centrifugation at $149,000 \times \mathrm{g}$ for $45 \mathrm{~min}$ and the solubilized protein was loaded onto a gravity column of $2 \mathrm{~mL} \mathrm{M} 2$ anti-FLAG affinity matrix (Sigma). The column was washed with $10 \mathrm{~mL}$ of wash buffer (50 mM Tris- $\mathrm{HCl} \mathrm{pH} 7.4,150 \mathrm{mM} \mathrm{NaCl}, 0.02 \%$ [w/v] DDM) and eluted with $5 \mathrm{~mL}$ of wash buffer supplemented with $3 \times$ FLAG peptide at $150 \mu \mathrm{g} / \mathrm{mL}$.

\section{Activity assays}

2,3-dimethyl-[1,4]naphthoquinol (DMW) (Enamine) at $20 \mathrm{mM}$ in anhydrous ethanol (400 $\mu \mathrm{L})$ on ice was reduced with a few grains of $\mathrm{NaBH}_{4}$ and the reaction was quenched by addition of $4 \mathrm{~N}$ $\mathrm{HCl}(10$ to $20 \mu \mathrm{L})$. Oxygen-reduction assays were performed with a Clark-type electrode (Oxygraph, Hansatech) in $1 \mathrm{~mL}$ of reaction buffer $(50 \mathrm{mM}$ Tris- $\mathrm{HCl} \mathrm{pH} 7.5,100 \mathrm{mM} \mathrm{NaCl}, 0.5$ mM EDTA, and $500 \mathrm{nM}$ bovine SOD [Sigma]). $\mathrm{CIII}_{2} \mathrm{CIV}_{2}$ was added (65 nM) and reactions were initiated by addition of $100 \mu \mathrm{M} \mathrm{DMWH}$. For inhibition studies, telacebec (DC Chemicals) at varying concentrations was incubated with $65 \mathrm{nM} \mathrm{CIII}_{2} \mathrm{CIV}_{2}$ in the reaction buffer for $3 \mathrm{~h}$ at 4 ${ }^{\circ} \mathrm{C}$. This mixture was added to the Oxygraph and reactions were initiated by addition of $100 \mu \mathrm{M}$ $\mathrm{DMWH}_{2}$. To account for any background oxygen reduction that still occurs in the presence of $\mathrm{SOD}$, the rate of oxygen reduction in the presence of $\mathrm{DMWH}_{2}$ and $\mathrm{SOD}$, but in the absence of $\mathrm{CIII}_{2} \mathrm{CIV}_{2}$, was subtracted from the rate in the presence of $\mathrm{DMWH}_{2}, \mathrm{SOD}$ and $\mathrm{CIII}_{2} \mathrm{CIV}_{2}$. The resulting oxygen reduction rates for $\mathrm{CIII}_{2} \mathrm{CIV}_{2}$ at different concentrations of telacebec were fit with a Python script. Individual inhibition curves, which were produced on different days with different preparations of reagent were fit individually, with the average of the $\mathrm{IC}_{50}$ values reported and the standard deviation of the fitted $\mathrm{IC}_{50}$ values reported as the error (Dahlin et al., 2004). Plots were produced using the Python matplotlib library. 


\section{CryoEM specimen preparation and imaging}

For cryoEM of inhibitor-free $\mathrm{CIII}_{2} \mathrm{CIV}_{2}$, enzyme at $\sim 16 \mathrm{mg} / \mathrm{mL}(2 \mu \mathrm{L})$ was applied to homemade nanofabricated holey gold grids (Marr et al., 2014), which had previously been glow-discharged in air for $120 \mathrm{~s}$ at $20 \mathrm{~mA}$ (PELCO easiGlow), within a Vitrobot Mark III (FEI) at $4{ }^{\circ} \mathrm{C}$ and 100 $\%$ relative humidity. Grids were blotted for $24 \mathrm{~s}$ before freezing. For cryoEM of telacebec-bound $\mathrm{CIII}_{2} \mathrm{CIV}_{2}, \mathrm{DMWH}_{2}$ in ethanol was added to $100 \mu \mathrm{M}(0.02 \%$ ethanol $)$ and telacebec in DMSO was added to $25 \mu \mathrm{M}\left(1.5 \%\right.$ DMSO) to a solution containing purified $\mathrm{CIII}_{2} \mathrm{CIV}_{2}$ at $\sim 0.08 \mathrm{mg} / \mathrm{mL}$ $(6 \mathrm{~mL})$. The solution was concentrated $\sim 100$-fold by centrifugation at $700 \times \mathrm{g}$ with a $100 \mathrm{kDa}$ molecular weight cutoff centrifuged concentrator device (Sigma). The sample $(2 \mu \mathrm{L})$ was then applied to homemade nanofabricated holey gold grids, which had previously been glowdischarged in air for $120 \mathrm{~s}$ at $20 \mathrm{~mA}$, within an EM GP2 (Leica) grid freezing device at $4{ }^{\circ} \mathrm{C}$ and $100 \%$ relative humidity. Grids were blotted for $1 \mathrm{~s}$ before freezing.

Screening of specimens was done with an FEI Tecnai F20 electron microscope equipped with a K2 Summit direct detector device camera. High-resolution cryoEM data were collected with a Titan Krios G3 electron microscope (Thermo Fisher Scientific) operated at $300 \mathrm{kV}$ and equipped with a Falcon 4 direct detector device camera. Automated data collection was done with the EPU software package. The inhibitor-free dataset consisted of 2,793 movies and telacebec-bound sample consisted of 4,308 movies. Movies were collected at a nominal magnification of 75,000× with a calibrated pixel size of $1.03 \AA$. Movies consisted of 30 exposure fractions over $7.7 \mathrm{~s}$. The camera exposure rate and the total exposure were $5.99 \mathrm{e}-/$ pixel $/ \mathrm{s}$ and $\sim 43.5 \mathrm{e}^{-/} \AA^{2}$, respectively (Table 1). Contrast transfer function estimation, particle selection, motion correction, map calculation, and three-dimensional variability analysis (3DVA) were all done within the cryoSPARC software package (Punjani et al., 2020, 2017; Punjani and Fleet, 2021; Rubinstein and Brubaker, 2015).

\section{Image analysis and atomic model building}

All image analysis was performed within the cryoSPARC software package, ver. 3 (Punjani et al., 2017), including individual particle motion correction (Rubinstein and Brubaker, 2015), nonuniform refinement (Punjani et al., 2020), and 3D Variability analysis (Punjani and Fleet, 2021). Image analysis and 3D reconstruction for each dataset was performed in the same way. Motion was corrected and contrast transfer function (CTF) parameters were estimated for each movie in patches. Manual particle selection and 2D classification was used to generate templates, which were in turn used to select of 387,777 and 1,037,709 particle images for the telacebec-bound and inhibitor-free datasets, respectively. Datasets were cleaned with 2D classification, 3D classification, and heterogeneous refinement to 70,818 and 150,885 particle images for the telacebec-bound and inhibitor-free datasets, respectively. Beam-tilt was corrected and each map was refined with non-uniform refinement without symmetry enforced. CTF values were then refined, the detergent micelle subtracted, and alignment parameters adjusted with local refinement with $\mathrm{C} 2$ symmetry enforced, yielding maps at $3.0 \AA$ resolution for each dataset. 3D variability analysis was done on a pooled dataset with masks including the SOD subunit or cyt. cc domain. Atomic models were constructed starting from previous models of the complex (Gong et al., 2018; Wiseman et al., 2018). Additions to the models were made in Coot (Emsley et al., 2010) and refined with Phenix (Liebschner et al., 2019) and ISOLDE (Croll, 2018). 


\section{Structure-Activity relation analysis studies}

Insight into protein-inhibitor interaction was facilitated by analysis with the Schrödinger software package (Release 2019-1). The protein preparation wizard within Schrödinger was used to prepare the protein for modelling. Briefly, the QcrA and QcrB chains and the ligand from the PDB file were merged and pre-processed to add missing hydrogen atoms, fill in missing side chains, and adjust ionization and tautomeric states of the ligand. The hydrogen bond network between the protein's amino acids and the ligand was optimized by allowing reorientation of amino acid side chains like His, Asn, Asp, Glu, and Gln and the ionization and tautomeric states of these side chains were estimated (Olsson et al., 2011). The resulting structure was refined to remove clashes and optimize geometry with the OPLSe* force field (Roos et al., 2019). These changes did not noticeably affect the fit of the model within the experimental cryoEM density map.

Competing interests: The authors declare no competing interests.

\section{Data availability}

Data deposition: all electron cryomicroscopy maps described in this article have been deposited in the Electron Microscopy Data Bank (EMDB) (accession numbers EMD-XXXX to EMD-XXXX) and atomic models have been deposited in the Protein Database (PDB) (accession numbers XXXX).

\section{Author contributions}

PB and JLR conceived the project and coordinated experiments. PB, PI, and JLR supervised the research. SAB prepared the M. smegmatis strain used for these experiments. DJY cultured cells, purified protein, prepared and imaged cryoEM specimens. DJY and SK performed enzyme assays. DJY and JDT performed cryoEM image analysis and atomic model building and refinement. JDT and RA analyzed the telacebec structure-activity relations. DJY, JDT, and JLR wrote the manuscript and prepared the figures with input from the other authors.

\section{Acknowledgements}

DJY was supported by a Canada Graduate Scholarship from the Canadian Institutes of Health Research (CIHR) and a Queen Elizabeth II Graduate Scholarship in Science and Technology from the University of Toronto Department of Medical Biophysics, JMDT was supported by a postdoctoral fellowship from the Canadian Institutes of Health Research, and JLR was supported by the Canada Research Chairs program. This research was supported by CIHR grant JT162186 (JLR), The Alice and Knut Wallenberg Foundation grant 2019.0043

(PB), and Swedish Research Council grant 2018-04619 (PB). Cryo-EM data were collected at the Toronto High-Resolution High-Throughput Cryo-EM facility, supported by the Canada Foundation for Innovation and Ontario Research Fund. 


\section{Figures}
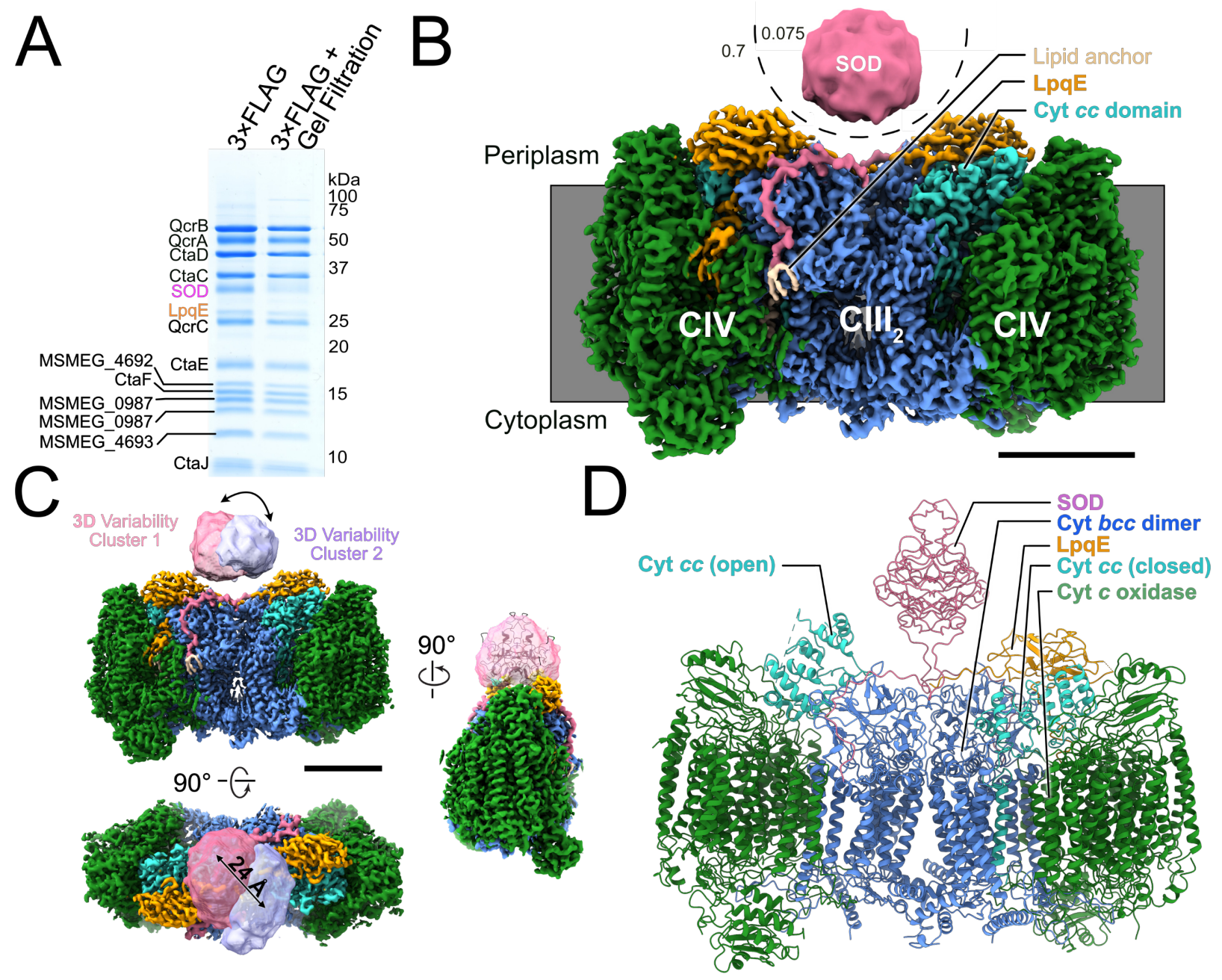

Figure 1. Structure of the $M$. smegmatis $\mathrm{CIII}_{2} \mathrm{CIV}_{2}$ respiratory complex. A, SDS-PAGE shows most of the known subunits of the complex and indicates that the SOD and LpqE subunits are depleted by gel filtration chromatography. B, CryoEM map of the $\mathrm{CIII}_{2} \mathrm{CIV}_{2}$. The different density thresholds for the SOD subunit and the rest of the complex are indicated. Scale bar, $50 \AA$. C, Three-dimensional variability analysis indicates two different clusters of particle images ("cluster 1" and "cluster 2") that show the SOD subunit in different positions over the 2-fold symmetry axis of the complex. Scale bar, $50 \AA$. D, An atomic model for the $\mathrm{CIII}_{2} \mathrm{CIV}_{2}$ complex with SOD fitted into the map and showing one half of the complex missing the LpqE subunit and with the cyt. $c c$ domain in the 'open' conformation and the other half of the complex possessing the LpqE subunit and with the cyt. $c c$ domain in the 'closed' conformation. 


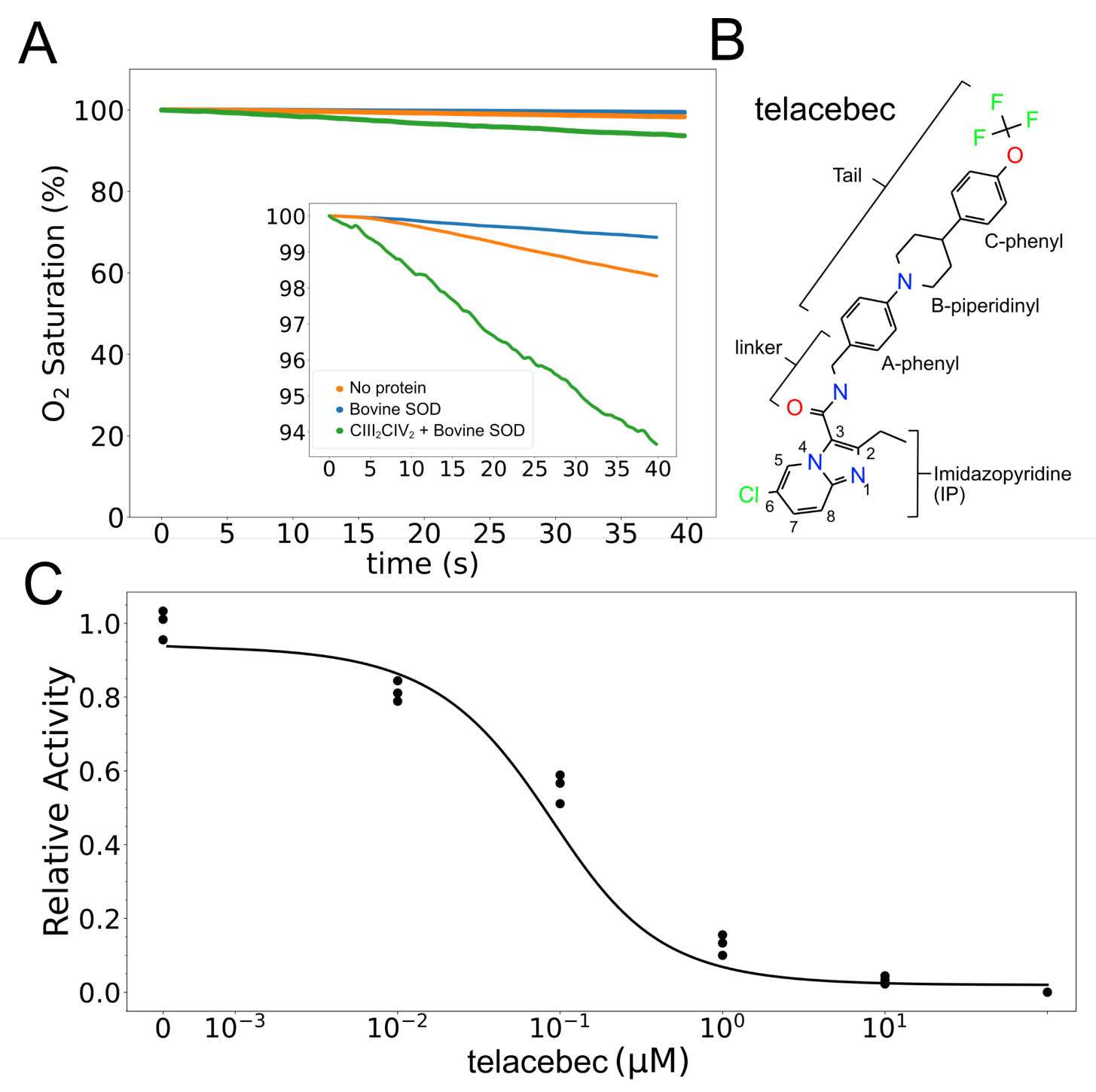

Figure 2. Assay of $\mathrm{DMWH}_{2}: \mathrm{O}_{2}$ oxidoreductase activity by $\mathrm{CIII}_{2} \mathrm{CIV}_{2}$. A, An oxygen reduction assay shows that autoxidation of 2,3-dimethyl-[1,4]naphthoquinone (DMW), blue curve, is decreased by the presence of $500 \mathrm{nM}$ bovine SOD, orange curve. Measurement of oxygen reduction by $\mathrm{CIII}_{2} \mathrm{CIV}_{2}$ in the presence of bovine SOD, green curve, allows calculation of $\mathrm{CIII}_{2} \mathrm{CIV}_{2}$ activity. B, Structure of $\mathrm{CIII}_{2} \mathrm{CIV}_{2}$ inhibitor telacebec (Q203). C, Titration of $\mathrm{CIII}_{2} \mathrm{CIV}_{2}(60 \mathrm{nM})$ with telacebec shows an $\mathrm{IC}_{50}$ of $53 \pm 19 \mathrm{nM}( \pm$ s.d., $\mathrm{n}=3$ independent titrations) with $100 \mu \mathrm{M} \mathrm{DMWH}$. 

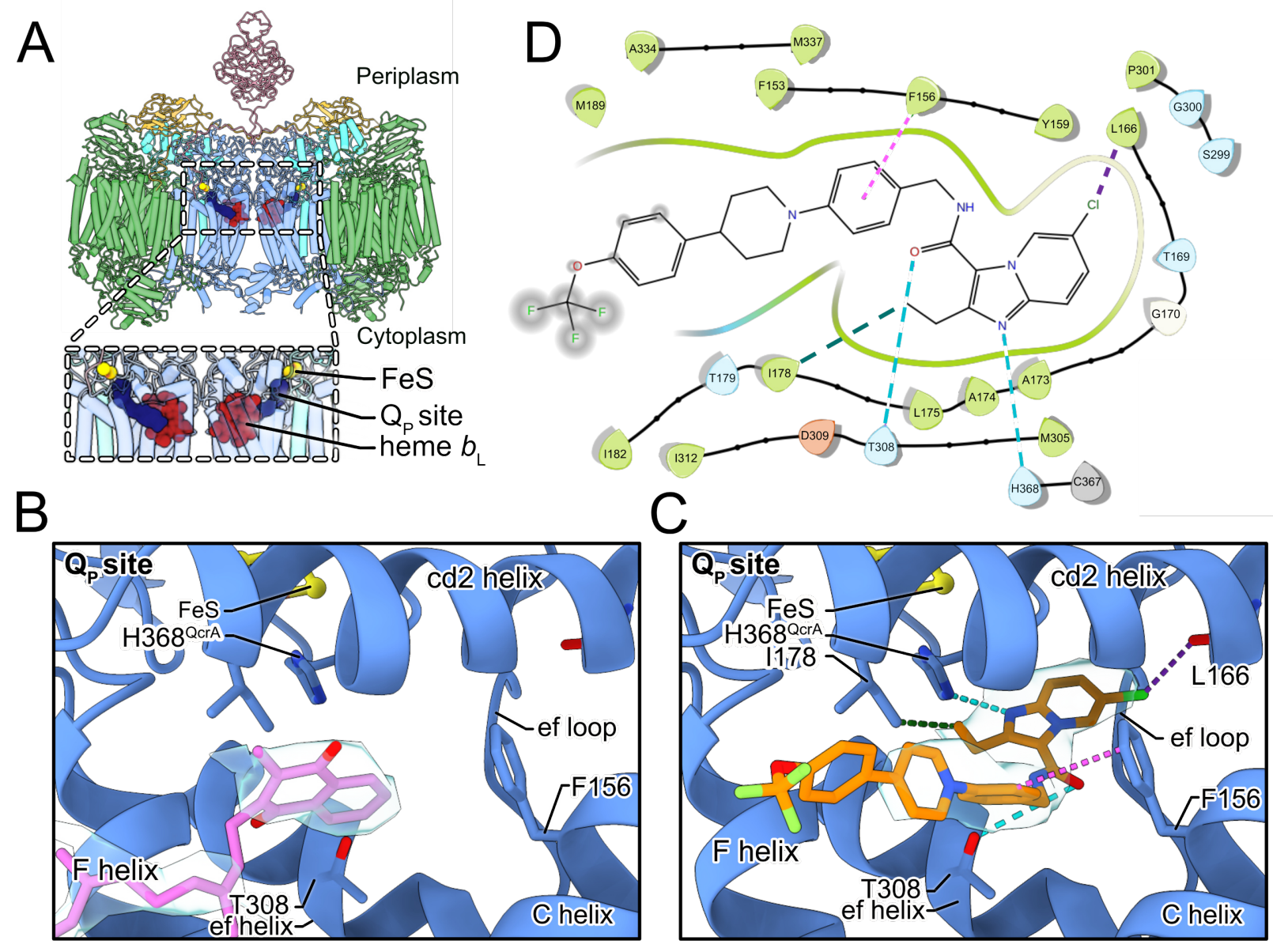

Figure 3. Telacebec binding to the $Q_{p}$ site. A, The dashed boxes indicate the two $Q_{p}$ sites in $\mathrm{CIII}_{2} \mathrm{CIV}_{2}$, each showing menaquinone (blue), the Rieske protein FeS (yellow), and heme $b_{L}$ (red). B, In the inhibitor-free structure, there is density for endogenous menaquinone (pink model and grey surface) distal from the FeS group amongst the well-conserved structural elements of the Qp site. C, In the inhibitor-bound structure there is density for telacebec (orange model and grey surface) deeper in the Qp site where it can form numerous interactions with the protein, including possible hydrogen bonds (dashed teal lines), hydrophobic interactions (dashed green line), a halogen bond (dashed purple line), and an aromatic interaction (dashed pink line). D, A $2 \mathrm{D}$ representation of the interactions between telacebec and residues of $\mathrm{CIII}_{2} \mathrm{CIV}_{2}$ using the same colour convention as in part $\mathrm{C}$. 


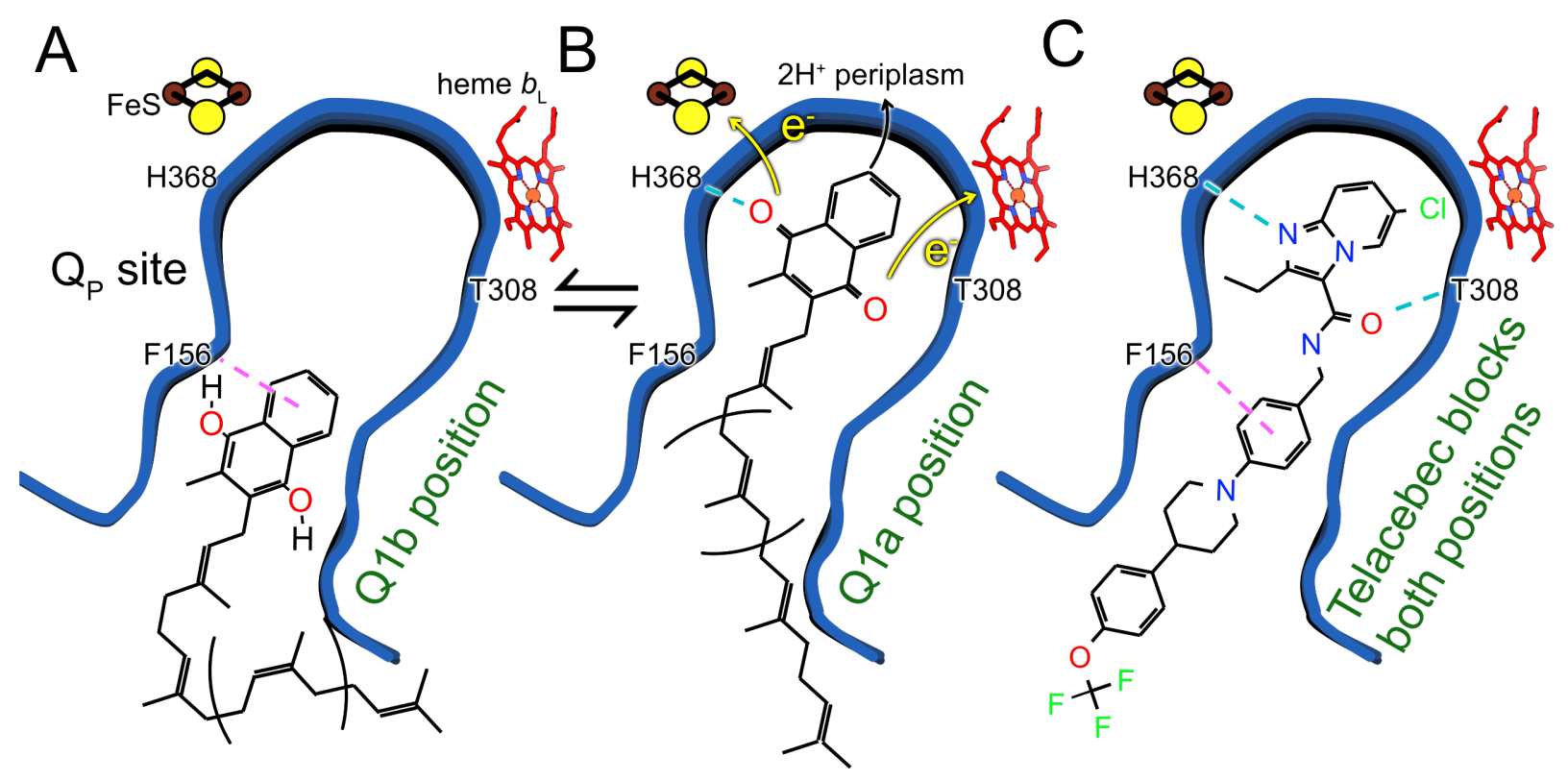

Figure 4. Model for oxidation of $\mathrm{MQH}_{2}$ in the $\mathrm{Q}_{\mathrm{P}}$ site and how telacebec blocks it. An emerging model for $\mathrm{MQH}_{2}$ reduction at the $\mathrm{Q}_{\mathrm{P}}$ site proposes that the substrate binds in the Q1b position where it is too far from FeS to donate protons and electrons (A). Upon moving deeper into the $\mathrm{Q}_{\mathrm{P}}$ site to the Q1a position, $\mathrm{MQH}_{2}$ is oxidized to $\mathrm{MQ}$, donating its first electron to FeS, its second electron to heme $b_{\mathrm{L}}$, and releasing two protons to the positively charged periplasmic side of the lipid bilayer (B). Telacebec binds deep within the QP site, forming numerous interactions with the protein and blocking both the Q1a and Q1b positions $(\mathbf{C})$. 
Figure Supplements

A Inhibitor-free
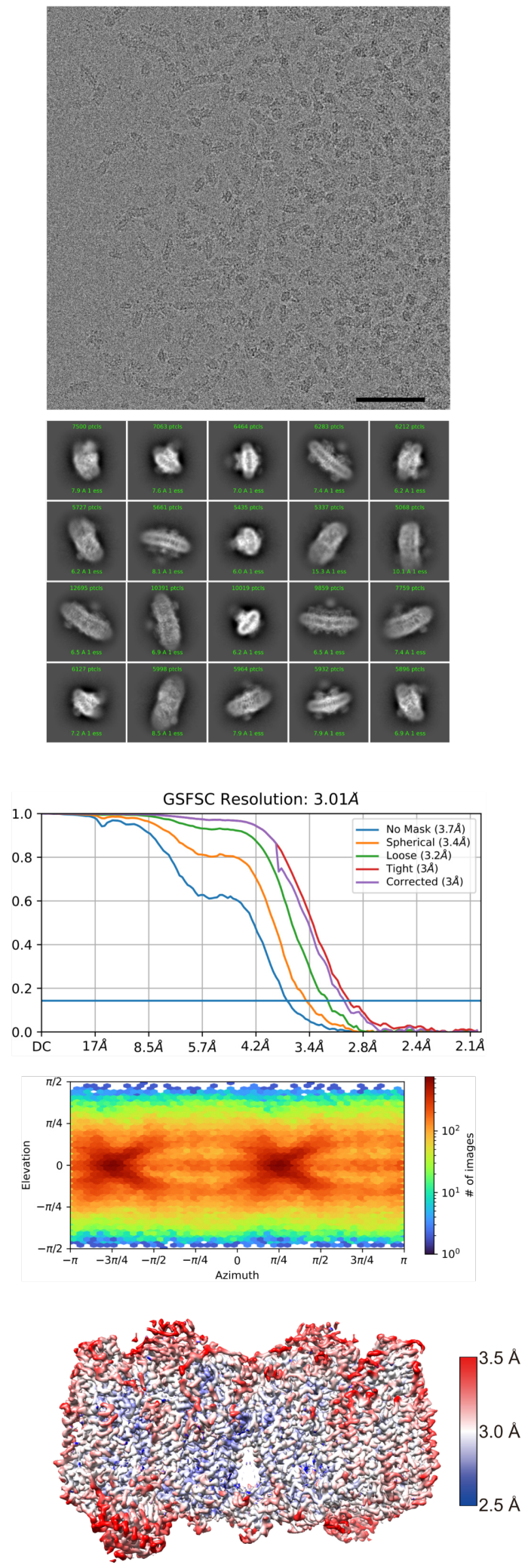

B Telacebec-bound
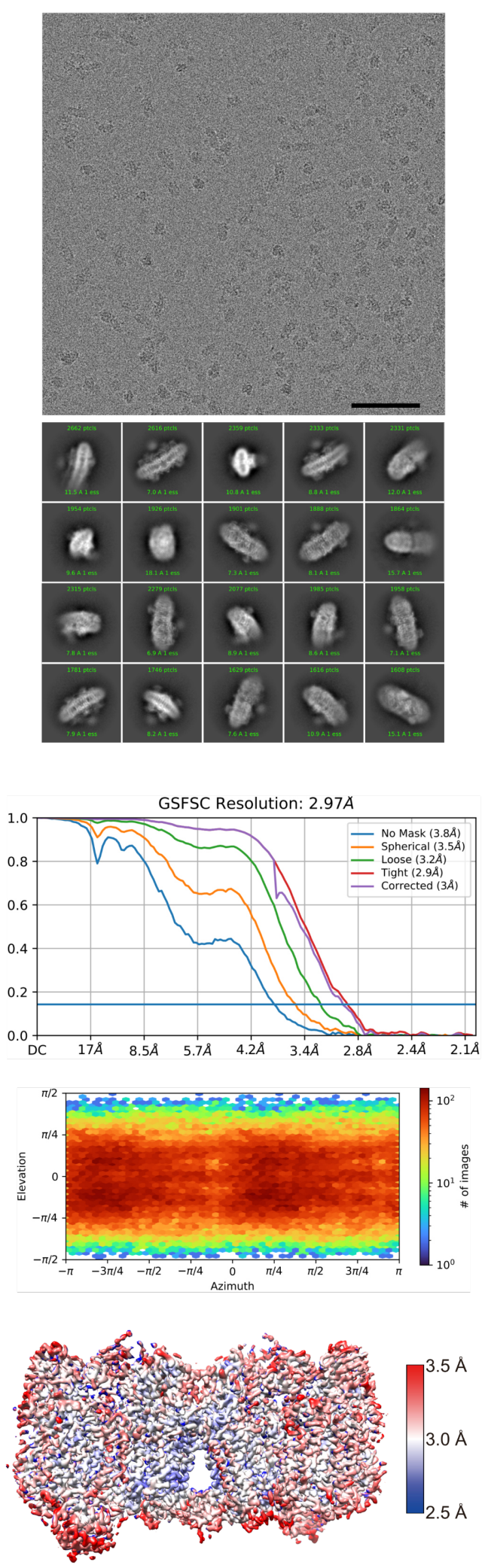
Figure 1 - Figure Supplement 1. CryoEM map validation. A, An example micrograph, class average images, Fourier Shell Correlation curves (including correction for the effects of masking following gold-standard refinement), orientation distribution plot, and local resolution estimate are shown for the inhibitor-free $\mathrm{CIII}_{2} \mathrm{CIV}_{2}$ map. B, An example micrograph, class average images, Fourier Shell Correlation curves (including correction for the effects of masking following gold-standard refinement), orientation distribution plot, and local resolution estimate are shown for the telacebec-bound $\mathrm{CIII}_{2} \mathrm{CIV}_{2}$ map. Scale bars, $50 \mathrm{~nm}$.
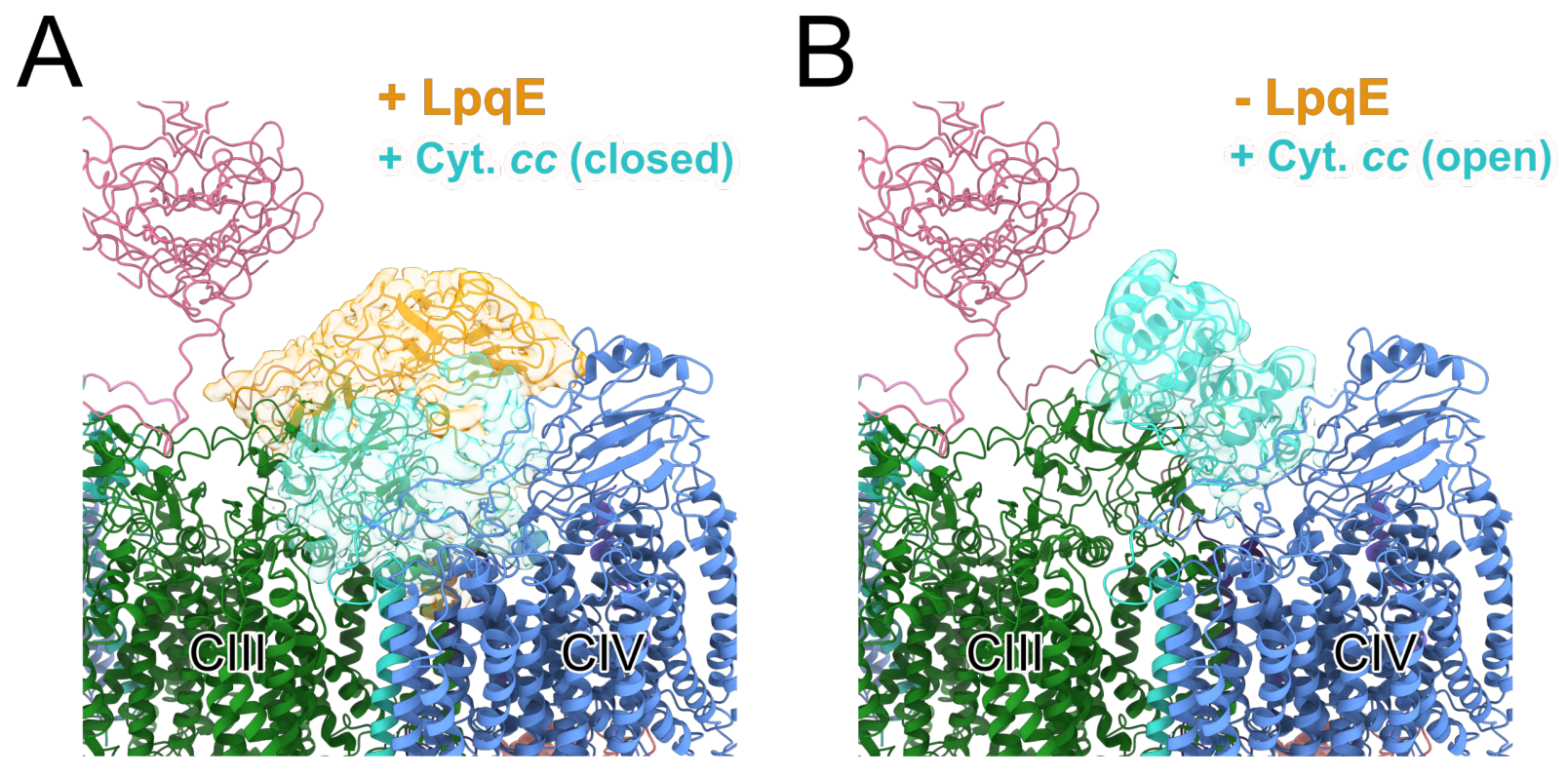

Figure 1 - Figure Supplement 2. 3D variability analysis of $\mathrm{LpqE}$ and the cyt. $c c$ domain. Focusing on one half of the $\mathrm{CIII}_{2} \mathrm{CIV}_{2}$ supercomplex with symmetry expansion to allow all half complexes to contribute, complexes were identified with LpqE (gold) and the cyt. $c c$ domain (cyan) in the 'closed' position (A) and without LpqE and the cyt. cc domain (cyan) in the 'open' position (B). 


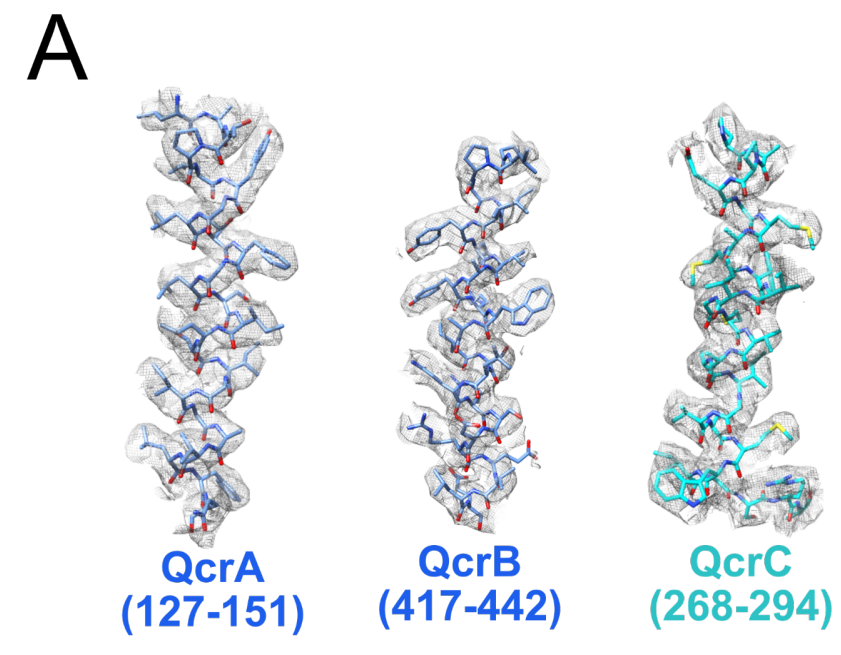

B

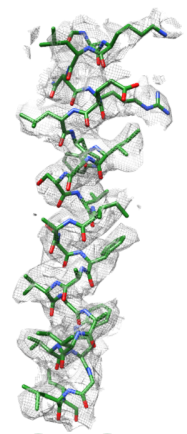

CtaC

(40-68)

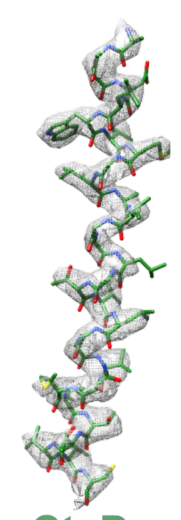

CtaD

(166-199)

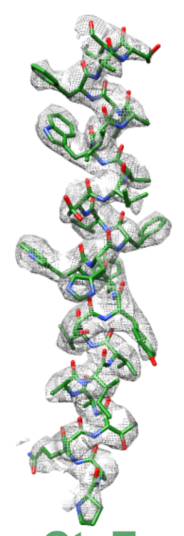

CtaE

(172-195)

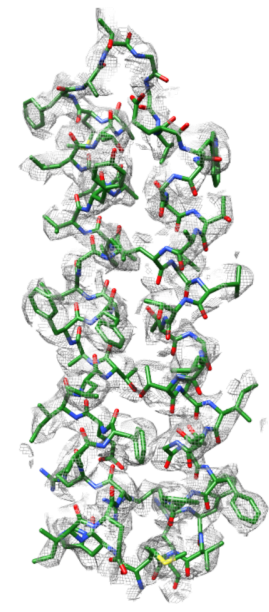

CtaF

(1-61)

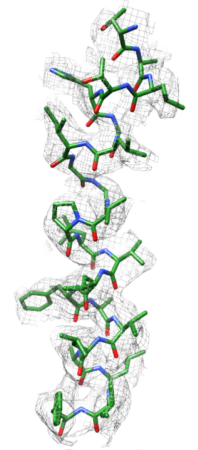

CtaJ

(3-25)

C

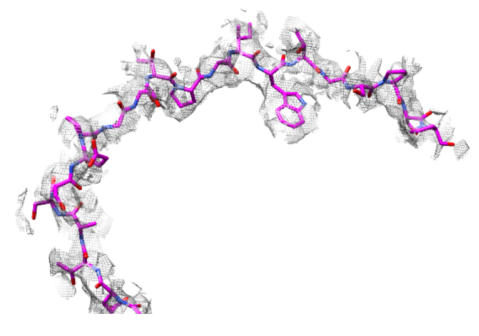

SOD

(25-42)
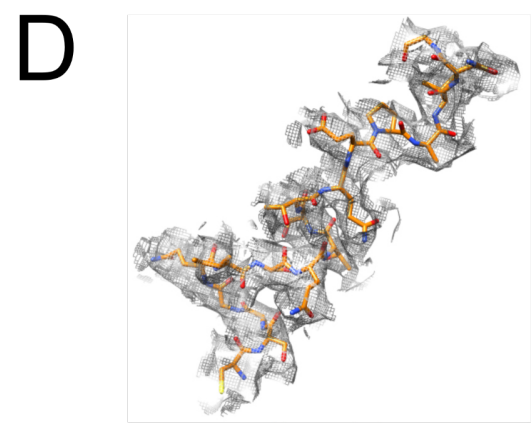

LpqE

(24-40)

Figure 1 - Figure Supplement 3. Examples of model in map fit. Examples for model in map fit are shown for the CIII part of the supercomplex (A), the CIV part of the supercomplex (B), as well as the SOD (C) and LpqE (D) subunits. 

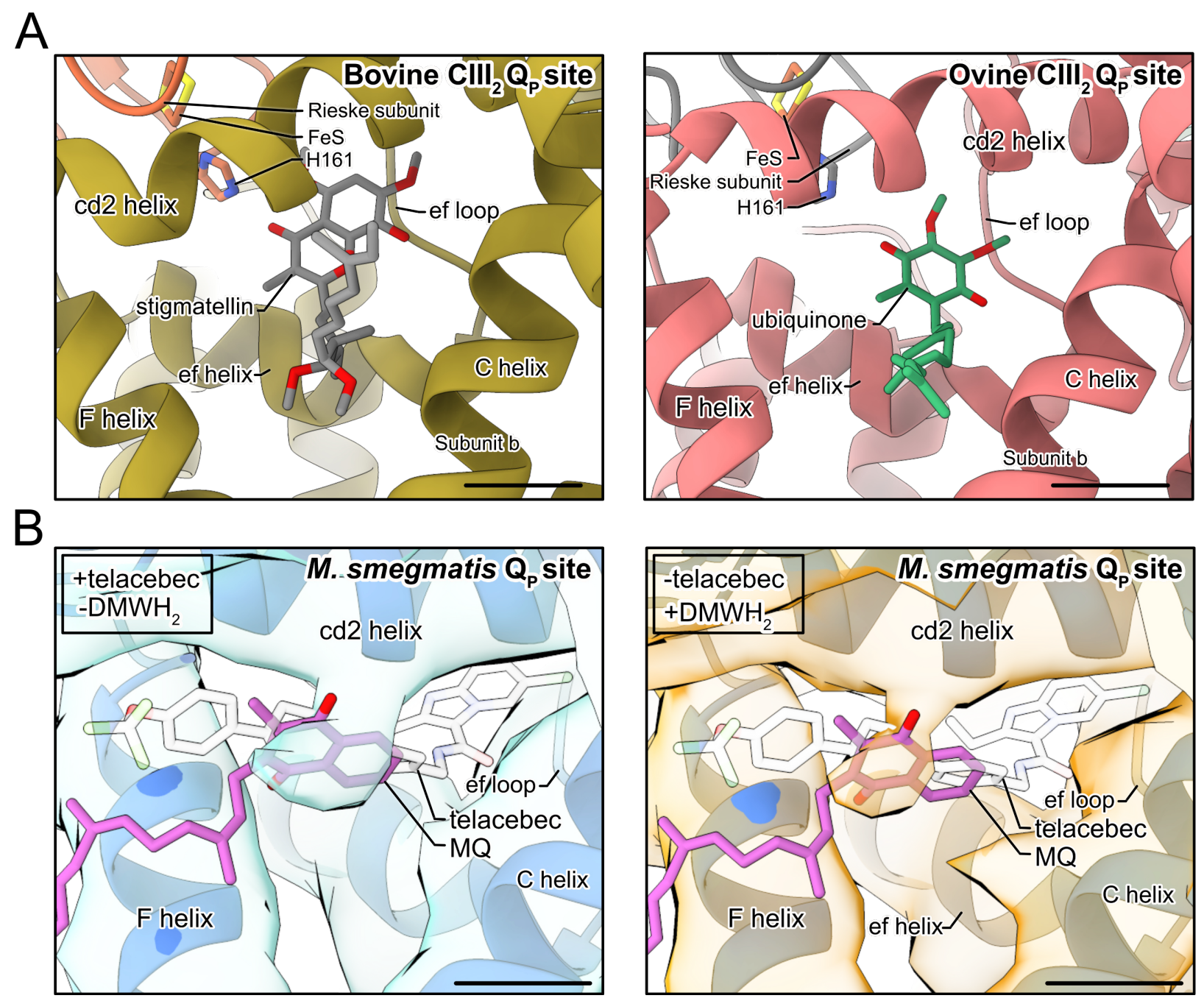

Figure 3 - Figure Supplement 1. Binding of small molecules in the QP site of CIII. A, Both stigmatellin (grey model) in the bovine $\mathrm{CIII}_{2} \mathrm{Q}_{\mathrm{P}}$ site (left) (PDB: 1PPJ) (Huang et al., 2005) and ubiquinone (green model) in the ovine $\mathrm{CICIII}_{2}$ supercomplex QP (right) (PDB: 6Q9E) (Letts et al., 2019) bind deep in the site close to the FeS group, different from endogenous MQ with $M$. smegmatis $\mathrm{CIII}_{2} \mathrm{CIV}_{2}$. B, CryoEM density from the $200 \mathrm{kV}$ screening microscope shows that $\mathrm{CIII}_{2} \mathrm{CIV}_{2}$ with telacebec but without $\mathrm{DMWH}_{2}$ at $4.7 \AA$ resolution (left) and with $\mathrm{DMWH}_{2}$ but without telacebec at $4.4 \AA$ resolution (right) is consistent with binding of MQ (pink model) but not telacebec (white model) in the Qp site. Scale bars, $5 \AA$. 
Table 1. CryoEM structure determination

A. Cryo-EM data acquisition and image processing.

\begin{tabular}{|l|l|}
\hline Data Collection & Titan Krios G3 \\
\hline Electron Microscope & Falcon 4 \\
\hline Camera & 300 \\
\hline Voltage $(\mathrm{kV})$ & 75,000 \\
\hline Nominal Magnification & 1.03 \\
\hline Calibrated pixel size $(\AA \AA)$ & 43.5 \\
\hline Total exposure $\left(\mathrm{e} / \AA^{2}\right)$ & 5.99 \\
\hline Exposure rate $(\mathrm{e} / \mathrm{pixel} / \mathrm{s})$ & 29 \\
\hline Number of exposure fractions & $0.7-2$ \\
\hline Defocus range $(\mu \mathrm{m})$ & \multicolumn{2}{|l|}{} \\
\hline Image Processing & cryoSPARC v3 \\
\hline Motion correction software & cryoSPARC v3 \\
\hline CTFestimation software & cryoSPARC v3 \\
\hline Particle selection software & 4,308 \\
\hline Micrographs used in inhibitor-free dataset & 2,793 \\
\hline Micrographs used in telacebec-bounddataset & $1,037,709$ \\
\hline Particle images selected in inhibitor free dataset & 387,777 \\
\hline Particle images selected in telacebec-bound dataset & cryoSPARC v3 \\
\hline 3D map classification and refinement software &
\end{tabular}

B. Model statistics.

\begin{tabular}{|l|l|l|}
\hline Dataset & Inhibitor-free & Telacebec-bound \\
\hline Associated PDB ID & & \\
\hline $\begin{array}{l}\text { Modelling and refinement } \\
\text { software }\end{array}$ & Coot, phenix, ISOLDE & Coot, phenix, ISOLDE \\
\hline Protein residues & 6,058 & 6,075 \\
\hline Ligand & $\begin{array}{l}\text { 9XX: 4, 9Y0: 6, 9YF: 8, FES: } \\
\text { 2, HEC: 4, HEA: 4, MQ9: } 10, \\
\text { HEM: 4, PLM: 4, CU: } 8\end{array}$ & $\begin{array}{l}\text { 9XX: 4, 9Y0: 6, 9YF: 8, FES: 2, } \\
\text { HEC: HEA: 4, MQ9: 10, } \\
\text { HEM: PLM: 4, CU: 8, QTE: } 1\end{array}$ \\
\hline RMSD bond length( $(\AA)$ & 0.005 & 0.006 \\
\hline RMSD bond angle( $\left(^{\circ}\right)$ & 1.485 & 1.63 \\
\hline Ramachandranoutliers (\%) & 0.22 & 0.52 \\
\hline Ramachandranfavoured (\%) & 92.58 & 92.67 \\
\hline Rotamer outliers (\%) & 0 & 0 \\
\hline Cash score & 19.29 & 25.01 \\
\hline MolProbabity score & 2.25 & 2.35 \\
\hline EMRinger score & 3.12 & 2.58 \\
\hline
\end{tabular}




\section{References}

Almeida D V., Converse PJ, Omansen TF, Tyagi S, Tasneen R, Kim J, Nuermberger EL. 2020. Telacebec for ultrashort treatment of Buruli ulcer in a mouse model. Antimicrob Agents Chemother 64:1-10. doi:10.1128/AAC.00259-20

Andries K, Verhasselt P, Guillemont J, Gohlmann HWH, Neefs J-M, Winkler H, Gestel J Van, Timmerman P, Zhu M, Lee E, Williams P, Chaffoy D de, Huitric E, Hoffner S, Cambau E, Truffot-Pernot C, Lounis N, Jarlier V. 2005. A diarylquinoline drug active on the ATP synthase of Mycobacterium tuberculosis. Science (80-) 307:223-7. doi:10.1126/science. 1106753

Arora K, Ochoa-Montaño B, Tsang PS, Blundell TL, Dawes SS, Mizrahi V, Bayliss T, Mackenzie CJ, Cleghorn LAT, Ray PC, Wyatt PG, Uh E, Lee J, Barry CE, Boshoff HI. 2014. Respiratory flexibility in response to inhibition of cytochrome $C$ oxidase in Mycobacterium tuberculosis. Antimicrob Agents Chemother 58:6962-6965. doi:10.1128/AAC.03486-14

Beites T, O’Brien K, Tiwari D, Engelhart CA, Walters S, Andrews J, Yang HJ, Sutphen ML, Weiner DM, Dayao EK, Zimmerman M, Prideaux B, Desai P V., Masquelin T, Via LE, Dartois V, Boshoff HI, Barry CE, Ehrt S, Schnappinger D. 2019. Plasticity of the Mycobacterium tuberculosis respiratory chain and its impact on tuberculosis drug development. Nat Commun 10:1-12. doi:10.1038/s41467-019-12956-2

Berney M, Cook GM. 2010. Unique flexibility in energy metabolism allows mycobacteria to combat starvation and hypoxia. PLoS One 5. doi:10.1371/journal.pone.0008614

Burley ASK, Petsko G a. 1985. Aromatic-Aromatic Interaction: A Mechanism of Protein Structure Stabilization. Science (80-) 229:23-28.

Cadenas E, Mira D, Brunmark A, Lind C, Segura-Aguilar J, Ernster L. 1988. Effect of superoxide dismutase on the autoxidation of various hydroquinones-A possible role of superoxide dismutase as a superoxide: Semiquinone oxidoreductase. Free Radic Biol Med 5:71-79. doi:10.1016/0891-5849(88)90032-9

Cole ST, Eiglmeier K, Parkhill J, James KD, Thomson NR, Wheeler PR, Honoré N, Garnier T, Churcher C, Harris D, Mungall K, Basham D, Brown D, Chillingworth T, Connor R, Davies RM, Devlin K, Duthoy S, Feltwell T, Fraser A, Hamlin N, Holroyd S, Hornsby T, Jagels K, Lacroix C, Maclean J, Moule S, Murphy L, Oliver K, Quail MA, Rajandream MA, Rutherford KM, Rutter S, Seeger K, Simon S, Simmonds M, Skelton J, Squares R, Squares S, Stevens K, Taylor K, Whitehead S, Woodward JR, Barrell BG. 2001. Massive gene decay in the leprosy bacillus. Nature 409:1007-1011. doi:10.1038/35059006

Cook GM, Hards K, Dunn E, Heikal A, Nakatani Y, Greening C, Crick DC, Fontes FL, Pethe K, Hasenoehrl E, Berney M. 2017. Oxidative Phosphorylation as a Target Space for Tuberculosis: Success, Caution, and Future Directions. Microbiol Spectr 5:1-22. doi:10.1128/microbiolspec.TBTB2-0014-2016

Croll TI. 2018. ISOLDE: A physically realistic environment for model building into lowresolution electron-density maps. Acta Crystallogr Sect D Struct Biol 74:519-530. doi: $10.1107 / \mathrm{S} 2059798318002425$

Dahlin JL, Sittampalam GS, Coussens NP, Devanarayan V, Weidner JR, Iversen PW, Haas J V., Bronson DD, Trask, Jr. OJ, Wiernicki TR, Kahl SD. 2004. Basic Guidelines for Reporting Non-Clinical Data. Assay Guid Man 1-47.

Demangel C, Stinear TP, Cole ST. 2009. Buruli ulcer: Reductive evolution enhances 
pathogenicity of Mycobacterium ulcerans. Nat Rev Microbiol 7:50-60. doi:10.1038/nrmicro2077

Ding H, Robertson DE, Daldal F, Dutton PL. 1992. Cytochrome bc1 complex [2Fe-2S] cluster and its interaction with ubiquinone and ubihydroquinone at the Qo site: a doubleoccupancy Qo site model. Biochemistry 31:3144-3158. doi:10.1021/bi00127a015

Emsley P, Lohkamp B, Scott W, Cowtan K. 2010. Features and development of Coot. Acta Crystallogr D66:486-501. doi:10.1107/S0907444910007493

Esser L, Quinn B, Li YF, Zhang M, Elberry M, Yu L, Yu CA, Xia D. 2004. Crystallographic studies of quinol oxidation site inhibitors: A modified classification of inhibitors for the cytochrome bc1complex. J Mol Biol 341:281-302. doi:10.1016/j.jmb.2004.05.065

Gao X, Wen X, Esser L, Quinn B, Yu L, Yu CA, Xia D. 2003. Structural basis for the quinone reduction in the bc1 complex: A comparative analysis of crystal structures of mitochondrial cytochrome bc1 with bound substrate and inhibitors at the Qi site. Biochemistry 42:90679080. doi:10.1021/bi0341814

Global Tuberculosis Report. 2020. . Geneva: World Health Organization.

Gong H, Li Jun, Xu A, Tang Y, Ji W, Gao R, Wang S, Yu L, Tian C, Li Jingwen, Yen HY, Lam SM, Shui G, Yang X, Sun Y, Li X, Jia M, Yang C, Jiang B, Lou Z, Robinson C V., Wong LL, Guddat LW, Sun F, Wang Q, Rao Z. 2018. An electron transfer path connects subunits of a mycobacterial respiratory supercomplex. Science (80-) 8923. doi:10.1126/science.aat8923

Graf S, Fedotovskaya O, Kao WC, Hunte C, Ädelroth P, Bott M, Von Ballmoos C, Brzezinski P. 2016. Rapid Electron Transfer within the III-IV Supercomplex in Corynebacterium glutamicum. Sci Rep 6:1-14. doi:10.1038/srep34098

Guo H, Courbon GM, Bueler SA, Mai J, Liu J, Rubinstein JL. 2021. Structure of mycobacterial ATP synthase with the TB drug bedaquiline. Nature 589:143-147. doi:10.1038/s41586020-3004-3

Huang L, Cobessi D, Tung EY, Berry EA. 2005. Binding of the Respiratory Chain Inhibitor Antimycin to the Mitochondrial bc 1 Complex : A New Crystal Structure Reveals an Altered Intramolecular Hydrogen-bonding Pattern. J Mol Biol 351:573-597. doi:10.1016/j.jmb.2005.05.053

Kao W, Kleinschroth T, Nitschke W, Baymann F, Neehaul Y, Hellwig P, Richers S, Vonck J, Bott M, Hunte C. 2016. Biochimica et Biophysica Acta The obligate respiratory supercomplex from Actinobacteria. BBA - Bioenerg 1857:1705-1714. doi:10.1016/j.bbabio.2016.07.009

Letts JA, Fiedorczuk K, Degliesposti G, Skehel M, Sazanov LA. 2019. Structures of Respiratory Supercomplex I+III2 Reveal Functional and Conformational Crosstalk. Mol Cell 75:11311146.e6. doi:10.1016/j.molcel.2019.07.022

Liebschner D, Afonine P V, Baker ML, Bunkoczi G, Chen VB, Croll TI, Hintze B, Hung L-W, Jain S, McCoy AJ, Moriarty NW, Oeffner RD, Poon BK, Prisant MG, Read RJ, Richardson JS, Richardson DC, Sammito MD, Sobolev O V, Stockwell DH, Terwilliger TC, Urzhumtsev AG, Videau LL, Williams CJ, Adams PD. 2019. Macromolecular structure determination using X-rays, neutrons and electrons: recent developments in Phenix. Acta Crystallogr D75:861-877. doi:10.1107/S2059798319011471

Lu P, Asseri AH, Kremer M, Maaskant J, Ummels R, Lill H, Bald D. 2018. The antimycobacterial activity of the cytochrome bcc inhibitor Q203 can be enhanced by smallmolecule inhibition of cytochrome bd. Sci Rep 8:1-7. doi:10.1038/s41598-018-20989-8 
Malone LA, Qian P, Mayneord GE, Hitchcock A, Farmer DA, Thompson RF, Swainsbury DJK, Ranson NA, Hunter CN, Johnson MP. 2019. Cryo-EM structure of the spinach cytochrome b6f complex at 3.6 Å resolution. Nature 575:535-539. doi:10.1038/s41586-019-1746-6

Marr CR, Benlekbir S, Rubinstein JL. 2014. Fabrication of carbon films with approximately 500 $\mathrm{nm}$ holes for cryo-EM with a direct detector device. J Struct Biol 185:42-47. doi:10.1016/j.jsb.2013.11.002

Matsoso LG, Kana BD, Crellin PK, Lea-smith DJ, Pelosi A, Powell D, Dawes SS, Rubin H, Coppel RL, Mizrahi V. 2005. Function of the Cytochrome. J Bacteriol 187:6300-6308. doi:10.1128/JB.187.18.6300

Moe A, Kovalova T, Król S, Yanofsky DJ, Bott M, Sjostrand D, Rubinstein JL, Hogbom M, Brzezinski P. 2021. The respiratory supercomplex from C. glutamicum. bioRxiv 2021.06.16. doi:https://doi.org/10.1101/2021.06.16.448340

Moser CC, Farid TA, Chobot SE, Dutton PL. 2006. Electron tunneling chains of mitochondria. Biochim Biophys Acta - Bioenerg 1757:1096-1109. doi:10.1016/j.bbabio.2006.04.015

Mulkidjanian AY. 2005. Ubiquinol oxidation in the cytochrome bc 1 complex: Reaction mechanism and prevention of short-circuiting. Biochim Biophys Acta 1709:5-34. doi:10.1016/j.bbabio.2005.03.009

Murphy KC, Nelson SJ, Nambi S, Papavinasasundaram K, Baer CE, Sassetti CM. 2018. ORBIT: a New Paradigm for Genetic Engineering of Mycobacterial Chromosomes. MBio 9:e0146718.

Olsson MHM, SØndergaard CR, Rostkowski M, Jensen JH. 2011. PROPKA3: Consistent treatment of internal and surface residues in empirical $\mathrm{p}$ K a predictions. J Chem Theory Comput 7:525-537. doi:10.1021/ct100578z

Pethe K, Bifani P, Jang J, Kang S, Park S, Ahn S, Jiricek J, Jung J, Jeon HK, Cechetto J, Christophe T, Lee H, Kempf M, Jackson M, Lenaerts AJ, Pham H, Jones V, Seo MJ, Kim YM, Seo M, Seo JJ, Park D, Ko Y, Choi I, Kim R, Kim SY, Lim S, Yim SA, Nam J, Kang H, Kwon H, Oh CT, Cho Y, Jang Y, Kim Junghwan, Chua A, Tan BH, Nanjundappa MB, Rao SPS, Barnes WS, Wintjens R, Walker JR, Alonso S, Lee S, Kim Jungjun, Oh S, Oh T, Nehrbass U, Han SJ, No Z, Lee J, Brodin P, Cho SN, Nam K, Kim Jaeseung. 2013. Discovery of Q203, a potent clinical candidate for the treatment of tuberculosis. Nat Med 19:1157-1160. doi:10.1038/nm.3262

Piddington DL, Fang FC, Laessig T, Cooper AM, Orme IM, Buchmeier NA. 2001. Cu,Zn superoxide dismutase of Mycobacterium tuberculosis contributes to survival in activated macrophages that are generating an oxidative burst. Infect Immun 69:4980-4987. doi:10.1128/IAI.69.8.4980-4987.2001

Preiss L, Langer JD, Yildiz Ö, Eckhardt-Strelau L, Guillemont JEG, Koul A, Meier T. 2015. Structure of the mycobacterial ATP synthase Fo rotor ring in complex with the anti-TB drug bedaquiline. Sci Adv 1:e1500106. doi:10.1126/sciadv.1500106

Punjani A, Fleet DJ. 2021. 3D variability analysis: Resolving continuous flexibility and discrete heterogeneity from single particle cryo-EM. J Struct Biol 213:107702. doi:10.1016/j.jsb.2021.107702

Punjani A, Rubinstein JL, Fleet DJ, Brubaker MA. 2017. cryoSPARC: Algorithms for rapid unsupervised cryo-EM structure determination. Nat Methods 14:290-296. doi:10.1038/nmeth.4169

Punjani A, Zhang H, Fleet DJ. 2020. Non-uniform refinement: adaptive regularization improves single-particle cryo-EM reconstruction. Nat Methods 17:1214-1221. doi:10.1038/s41592- 
020-00990-8

Roos K, Wu C, Damm W, Reboul M, Stevenson JM, Lu C, Dahlgren MK, Mondal S, Chen W, Wang L, Abel R, Friesner RA, Harder ED. 2019. OPLS3e: Extending Force Field Coverage for Drug-Like Small Molecules. J Chem Theory Comput 15:1863-1874. doi:10.1021/acs.jctc.8b01026

Rubinstein JL, Brubaker MA. 2015. Alignment of cryo-EM movies of individual particles by optimization of image translations. J Struct Biol 192:1-11. doi:10.1016/j.jsb.2015.08.007

Rybniker J, Vocat A, Sala C, Busso P, Pojer F, Benjak A, Cole ST. 2015. Lansoprazole is an antituberculous prodrug targeting cytochrome bc1. Nat Commun 6:1-8. doi: $10.1038 /$ ncomms 8659

Sarewicz M, Osyczka A. 2015. Electronic connection between the quinone and cytochrome c redox pools and its role in regulation of mitochondrial electron transport and redox signaling. Physiol Rev 95:219-243. doi:10.1152/physrev.00006.2014

Van Der Werf TS, Barogui YT, Converse PJ, Phillips RO, Stienstra Y. 2020. Pharmacologic management of Mycobacterium ulcerans infection. Expert Rev Clin Pharmacol 13:391401. doi:10.1080/17512433.2020.1752663

van Niekerk C, Hutchings J, Kim J, Vanker N, van der Merwe L, Choi J, Nam K, Diacon. 2020. Telacebec (Q203), a New Antitubercular Agent. N Engl J Med 382:1280-1281. doi:10.1056/nejmc2001899

Vauquelin G, Charlton SJ. 2010. Long-lasting target binding and rebinding as mechanisms to prolong in vivo drug action. Br J Pharmacol 161:488-508. doi:10.1111/j.14765381.2010.00936.x

Wiseman B, Nitharwal RG, Fedotovskaya O, Schäfer J, Guo H, Kuang Q, Benlekbir S, Sjöstrand D, Ädelroth P, Rubinstein JL, Brzezinski P, Högbom M. 2018. Structure of a functional obligate respiratory supercomplex from Mycobacterium smegmatis. Nat Struct Mol Biol 25:1128-1136.

World Health Organization. 2019. The Selection and Use of Essential Medicines. Geneva: World Health Organization.

Xia D, Esser L, Tang WK, Zhou F, Zhou Y, Yu L, Yu CA. 2013. Structural analysis of cytochrome bc1 complexes: Implications to the mechanism of function. Biochim Biophys Acta - Bioenerg. doi:10.1016/j.bbabio.2012.11.008

Zhang Z, Huang L, Shulmeister VM, Chi YI, Kim KK, Hung LW, Crofts AR, Berry EA, Kim SH. 1998. Electron transfer by domain movement in cytochrome bc1. Nature 392:677-684. doi: $10.1038 / 33612$ 